\title{
Large-scale Parallel Computing of Cloud Resolving Storm Simulator
}

\author{
Kazuhisa Tsuboki ${ }^{1}$ and Atsushi Sakakibara ${ }^{2}$ \\ 1 Hydrospheric Atmospheric Research Center, Nagoya University \\ Furo-cho, Chikusa-ku, Nagoya, 464-8601 JAPAN \\ phone: +81-52-789-3493, fax: +81-52-789-3436 \\ e-mail: tsuboki@ihas.nagoya-u.ac.jp \\ 2 Research Organization for Information Science and Technology \\ Furo-cho, Chikusa-ku, Nagoya, 464-8601 JAPAN \\ phone: +81-52-789-3493, fax: +81-52-789-3436 \\ e-mail: atsusi@ihas.nagoya-u.ac.jp
}

\begin{abstract}
A sever thunderstorm is composed of strong convective clouds. In order to perform a simulation of this type of storms, a very fine-grid system is necessary to resolve individual convective clouds within a large domain. Since convective clouds are highly complicated systems of the cloud dynamics and microphysics, it is required to formulate detailed cloud physical processes as well as the fluid dynamics. A huge memory and large-scale parallel computing are necessary for the computation. For this type of computations, we have developed a cloud resolving numerical model which was named the Cloud Resolving Storm Simulator (CReSS). In this paper, we will describe the basic formulations and characteristics of CReSS in detail. We also show some results of numerical experiments of storms obtained by a large-scale parallel computation using CReSS.
\end{abstract}

\section{Introduction}

A numerical cloud model is indispensable for both understanding cloud and precipitation and their forecasting. Convective clouds and their organized storms are highly complicated systems determined by the fluid dynamics and cloud microphysics. In order to simulate evolution of a convective cloud storm, calculation should be performed in a large domain with a very high resolution grid to resolve individual clouds. It is also required to formulate accurately cloud physical processes as well as the fluid dynamic and thermodynamic processes. A detailed formulation of cloud physics requires many prognostic variables even in a bulk method such as cloud, rain, ice, snow, hail and so on. Consequently, a large-scale parallel computing with a huge memory is necessary for this type of simulation.

Cloud models have been developed and used for study of cloud dynamics and cloud microphysics since 1970's (e.g., Klemp and Wilhelmson, 1978 [1]; Ikawa, 1988 [2]; Ikawa and Saito, 1991 [3]; Xue et al., 1995 [4]; Grell et al., 1994 [5]). These models employed the non-hydrostatic and compressible equations systems 
with a fine-grid system. Since the computation of cloud models was very large, they have been used for research with a limited domain.

The recent progress in high performance computer, especially a parallel computers is extending the potential of cloud models widely. It enables us to perform a simulation of mesoscale storm using a cloud model. For this four years, we have developed a cloud resolving numerical model which was designed for parallel computers including "the Earth Simulator".

The purposes of this study are to develop the cloud resolving model and its parallel computing to simulate convective clouds and their organized storms. Thunderstorms which are organization of convective clouds produce many types of severe weather: heavy rain, hail storm, downburst, tornado and so on. The simulation of thunderstorms will clarify the characteristics of dynamics and evolution and will contribute to the mesoscale storm prediction.

The cloud resolving model which we are now developing was named "the Cloud Resolving Storm Simulator (CReSS)". In this paper, we will describe the basic formulation and characteristics of CReSS in detail. Some results of numerical experiments using CReSS will be also presented.

\section{Description of CReSS}

\subsection{Basic Equations and Characteristics}

The coordinate system of CReSS is the Cartesian coordinates in horizontal $x, y$ and a terrain-following curvilinear coordinate in vertical $\zeta$ to include the effect of orography. Using height of the model surface $z_{s}(x, y)$ and top height $z_{t}$, the vertical coordinate $\zeta(x, y, z)$ is defined as,

$$
\zeta(x, y, z)=\frac{z_{t}\left[z-z_{s}(x, y)\right]}{z_{t}-z_{s}(x, y)} .
$$

If we use a vertically stretching grid, the effect will be included in (1). Computation of CReSS is performed in the rectangular linear coordinate transformed from the curvilinear coordinate. The transformed velocity vector will be

$$
\begin{aligned}
U & =u, \\
V & =v \\
W & =\left(u J_{31}+v J_{32}+w\right) / G^{\frac{1}{2}} .
\end{aligned}
$$

where variable components of the transform matrix are defined as

$$
\begin{aligned}
J_{31} & =-\frac{\partial z}{\partial x}=\left(\frac{\zeta}{z_{t}}-1\right) \frac{\partial z_{s}(x, y)}{\partial x} \\
J_{32} & =-\frac{\partial z}{\partial y}=\left(\frac{\zeta}{z_{t}}-1\right) \frac{\partial z_{s}(x, y)}{\partial y} \\
J_{d} & =\frac{\partial z}{\partial \zeta}=1-\frac{z_{s}(x, y)}{z_{t}}
\end{aligned}
$$


and the Jacobian of the transformation is

$$
G^{\frac{1}{2}}=\left|J_{d}\right|=J_{d}
$$

In this coordinate, the governing equations of dynamics in CReSS will be formulated as follows. The dependent variables of dynamics are three-dimensional velocity components $u, v$ and $w$, perturbation pressure $p^{\prime}$ and perturbation of potential temperature $\theta^{\prime}$. For convenience, we use the following variables to express the equations.

$$
\begin{gathered}
\rho^{*}=G^{\frac{1}{2}} \bar{\rho}, \quad u^{*}=\rho^{*} u, \quad v^{*}=\rho^{*} v, \\
w^{*}=\rho^{*} w, \quad W^{*}=\rho^{*} W, \quad \theta^{*}=\rho^{*} \theta^{\prime} .
\end{gathered}
$$

where $\bar{\rho}$ is the density of the basic field which is in the hydrostatic balance.

Using these variables, the momentum equations are

$$
\begin{aligned}
& \frac{\partial u^{*}}{\partial t}=\underbrace{-\left(u^{*} \frac{\partial u}{\partial x}+v^{*} \frac{\partial u}{\partial y}+W^{*} \frac{\partial u}{\partial \zeta}\right)}_{[\mathbf{r m}]} \\
& \underbrace{-\left[\frac{\partial}{\partial x}\left\{J_{d}\left(p^{\prime}-\alpha D i v^{*}\right)\right\}+\frac{\partial}{\partial \zeta}\left\{J_{31}\left(p^{\prime}-\alpha D_{i v}^{*}\right)\right\}\right]}_{[\mathbf{a m}]} \\
& +\underbrace{\left(f_{s} v^{*}-f_{c} w^{*}\right)}_{[\mathbf{r m}]}+\underbrace{G^{\frac{1}{2}} \operatorname{Turb} . u}_{[\mathbf{p h y s i c s}]} \\
& \frac{\partial v^{*}}{\partial t}=\underbrace{-\left(u^{*} \frac{\partial v}{\partial x}+v^{*} \frac{\partial v}{\partial y}+W^{*} \frac{\partial v}{\partial \zeta}\right)}_{[\mathbf{r m}]} \\
& \underbrace{-\left[\frac{\partial}{\partial y}\left\{J_{d}\left(p^{\prime}-\alpha D i v^{*}\right)\right\}+\frac{\partial}{\partial \zeta}\left\{J_{32}\left(p^{\prime}-\alpha D i v^{*}\right)\right\}\right]}_{[\mathbf{a m}]} \\
& -\underbrace{f_{s} u^{*}}_{[\mathbf{r m}]}+\underbrace{G^{\frac{1}{2}} \text { Turb.v }}_{[\text {physics }]} \\
& \frac{\partial w^{*}}{\partial t}=\underbrace{-\left(u^{*} \frac{\partial w}{\partial x}+v^{*} \frac{\partial w}{\partial y}+W^{*} \frac{\partial w}{\partial \zeta}\right)}_{[\mathbf{r m}]} \underbrace{-\frac{\partial}{\partial \zeta}\left(p^{\prime}-\alpha D i v^{*}\right)}_{[\mathbf{a m}]} \\
& -\rho^{*} g(\underbrace{\frac{\theta^{\prime}}{\bar{\theta}}}_{[\mathbf{g m}]}-\underbrace{\frac{p^{\prime}}{\bar{\rho} c_{s}^{2}}}_{[\mathbf{a m}]}+\underbrace{\frac{q_{v}^{\prime}}{\epsilon+\bar{q}_{v}}-\frac{q_{v}^{\prime}+\sum q_{x}}{1+\bar{q}_{v}}}_{[\mathbf{p h y s i c s}]}) \\
& +\underbrace{f_{c} u^{*}}_{[\mathbf{r m}]}+\underbrace{G^{\frac{1}{2}} \text { Turb.w }}_{[\mathbf{p h y s i c s}]},
\end{aligned}
$$


where $\alpha D i v^{*}$ is an artificial divergence damping term to suppress acoustic waves, $f_{s}$ and $f_{c}$ are Coriolis terms, $c_{s}^{2}$ is square of the acoustic wave speed, $q_{v}$ and $q_{x}$ is mixing ratios of water vapor and hydrometeors, respectively. The equation of the potential temperature is

$$
\begin{aligned}
\frac{\partial \theta^{*}}{\partial t}= & \underbrace{-\left(u^{*} \frac{\partial \theta^{\prime}}{\partial x}+v^{*} \frac{\partial \theta^{\prime}}{\partial y}+W^{*} \frac{\partial \theta^{\prime}}{\partial \zeta}\right)}_{[\mathbf{r m}]} \\
& -\underbrace{\bar{\rho} w \frac{\partial \bar{\theta}}{\partial \zeta}}_{[\mathbf{g m}]}+\underbrace{G^{\frac{1}{2}} \operatorname{Turb} . \theta}_{[\text {physics }]}+\underbrace{\rho^{*} \text { Src. } \theta}_{[\text {physics }]}
\end{aligned}
$$

and the pressure equation is

$$
\begin{aligned}
\frac{\partial G^{\frac{1}{2}} p^{\prime}}{\partial t}= & \underbrace{-\left(J_{3} u \frac{\partial p^{\prime}}{\partial x}+J_{3} v \frac{\partial p^{\prime}}{\partial y}+J_{3} W \frac{\partial p^{\prime}}{\partial \zeta}\right)}_{[\mathbf{r m}]}+\underbrace{G^{\frac{1}{2}} \bar{\rho} g w}_{[\mathbf{a m}]} \\
& -\underbrace{\bar{\rho} c_{s}^{2}\left(\frac{\partial J_{3} u}{\partial x}+\frac{\partial J_{3} v}{\partial y}+\frac{\partial J_{3} W}{\partial \zeta}\right)}_{[\mathbf{a m}]}+\underbrace{G^{\frac{1}{2}} \bar{\rho} c_{s}^{2}\left(\frac{1}{\theta} \frac{d \theta}{d t}-\frac{1}{Q} \frac{d Q}{d t}\right)}_{[\mathbf{a m}]},
\end{aligned}
$$

where $Q$ is diabatic heating, terms of "Turb." is a physical process of the turbulent mixing and term of "Src." is source term of potential temperature.

Since the governing equations have no approximation, they will express all type of waves including the acoustic waves, gravity waves and Rossby waves. These waves have very wide range of phase speed. The fastest wave is the acoustic wave. Although it is unimportant in meteorology, its speed is very large in comparison with other waves and limits the time increment of integration. We, therefore, integrate the terms related to the acoustic waves and other terms with different time increments. In the equations $(9) \sim(13),[\mathbf{r m}]$ is indicates terms which are related to rotational mode (the Rossby wave mode), [gm] the divergence mode (gravity wave mode), and [am] the acoustic wave mode, respectively. Terms of physical processes are indicated by [physics].

\subsection{Computational Scheme and Parallel Processing Strategy}

In numerical computation, a finite difference method is used for the spatial discretization. The coordinates are rectangular and dependent variables are set on a staggered grid: the Arakawa-C grid in horizontal and the Lorenz grid in vertical (Fig.1). The coordinates $x, y$ and $\zeta$ are defined at the faces of the grid boxes. The velocity components $u, v$ and $w$ are defined at the same points of the coordinates $x, y$ and $\zeta$, respectively. The metric tensor $J_{31}$ is evaluated at a half interval below the $u$ point and $J_{32}$ at a half interval below the $v$ point. All scalar variables $p^{\prime}, \theta^{\prime}, q_{v}$ and $q_{x}$, the metric tensor $J_{d}$ and the transform Jacobian are 
defined at the center of the grid boxes. In the computation, an averaging operator is used to evaluate dependent variables at the same points. All output variables are obtained at the scalar points.

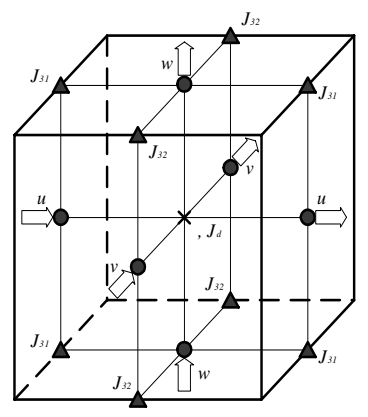

Fig. 1. Structure of the staggered grid and setting of dependent variables.

As mentioned in the previous sub-section, the governing equation includes all types of waves and the acoustic waves severely limits the time increment. In order to avoid this difficulty, CReSS adopted the mode-splitting technique (Klemp and Wilhelmson, 1978 [1]) for time integration. In this technique, the terms related to the acoustic waves in $(9) \sim(13)$ are integrated with a small time increment $\Delta \tau$ and all other terms are with a large time increment $\Delta t$.

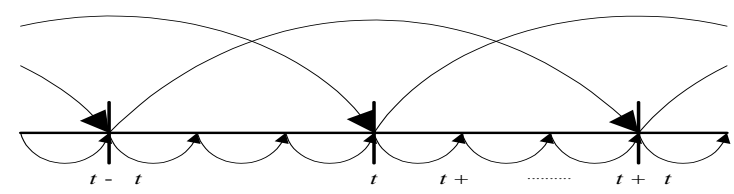

Fig. 2. Schematic representation of the mode-splitting time integration method. The large time step is indicated by the upper large curved arrows with the increment of $\Delta t$ and the small time step by the lower small curved arrows with the increment of $\Delta \tau$.

Figure 2 shows a schematic representation of the time integration of the mode-splitting technique. CReSS has two options in the small time step integration; one is an explicit time integration both in horizontal and vertical and the other is explicit in horizontal and implicit in vertical. In the latter option, $p^{\prime}$ and $w$ are solved implicitly by the Crank-Nicolson scheme in vertical. With respect to the large time step integration, the leap-frog scheme with the Asselin time filter is used for time integration. In order to remove grid-scale noise, the second or forth order computational mixing is used. 


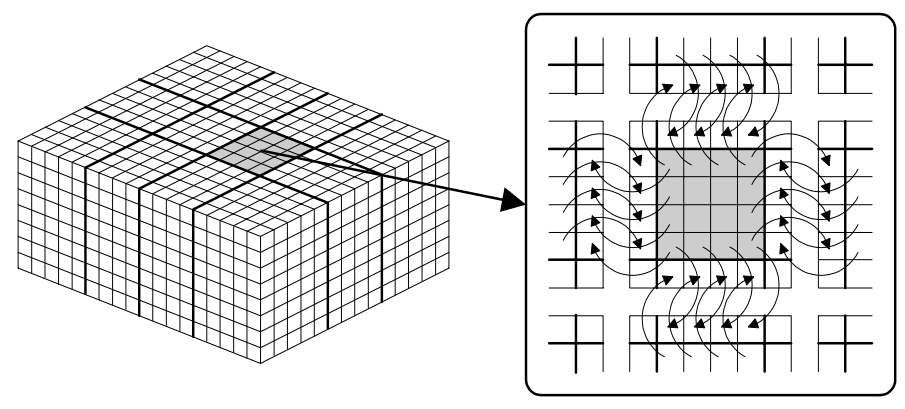

Fig. 3. Schematic representation of the two-dimensional domain decomposition and the communication strategy for the parallel computing.

A large three-dimensional computational domain (order of $100 \mathrm{~km}$ ) is necessary for the simulation of thunderstorm with a very high resolution (order of less than $1 \mathrm{~km}$ ). For parallel computing of this type of computation, CReSS adopts a two dimensional domain decomposition in horizontal (Fig.3). Parallel processing is performed by the Massage Passing Interface (MPI). Communications between the individual processing elements (PEs) are performed by data exchange of the outermost two grids.

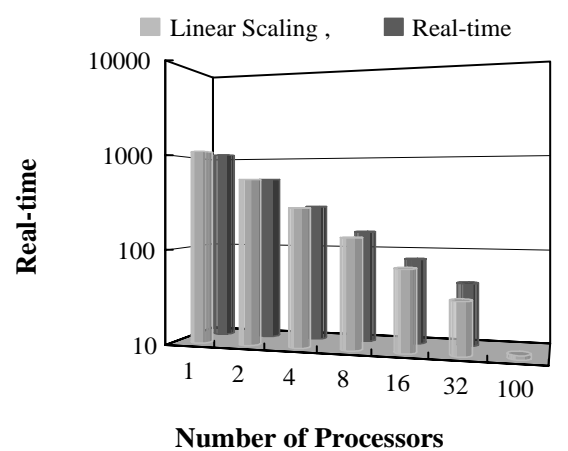

Fig. 4. Computation time of parallel processing of a test experiment. The model used in the test had $67 \times 67 \times 35$ grid points and was integrated for 50 steps on HITACHI SR2201.

The performance of parallel processing of CReSS was tested by a simulation whose grid size was $67 \times 67 \times 35$ on HITACHI SR2201. With increase of the number of PEs, the computation time decreased almost linearly (Fig.4). The efficiency was almost 0.9 or more if the number of PEs was less than 32. When the number of PEs was 32, the efficiency decreased significantly. Because the number of grid was too small to use the 32 PEs. The communication between 
PEs became relatively large. The results of the test showed a sufficiently high performance of the parallel computing of CReSS.

\subsection{Initial and Boundary Conditions}

Several types of initial and boundary conditions are optional in CReSS. For a numerical experiment, a horizontally uniform initial field provided by a sounding profile will be used with an initial disturbance of a thermal bubble or random noise. Optional boundary conditions are rigid wall, periodic, zero normalgradient, and wave-radiation type of Orlanski (1976) [6].

CReSS has an option to be nested within a coarse-grid model and performs a prediction experiment. In this option, the initial field is provided by interpolation of grid point values and the boundary condition is provided by the coarse-grid model.

\subsection{Physical Processes}

Cloud physics is an important physical process. It is formulated by a bulk method of cold rain which is based on Lin et al. (1983) [7], Cotton et al. (1986) [8], Murakami (1990) [9], Ikawa and Saito (1991) [3], and Murakami et al. (1994) [10]. The bulk parameterization of cold rain considers water vapor, rain, cloud, ice, snow, and graupel. Prognostic variables are mixing ratios for water vapor $q_{v}$, cloud water $q_{c}$, rain water $q_{r}$, cloud ice $q_{i}$, snow $q_{s}$ and graupel $q_{g}$. The prognostic equations of these variables are

$$
\begin{aligned}
& \frac{\partial \bar{\rho} q_{v}}{\partial t}=\text { Adv } \cdot q_{v}+\text { Turb } . q_{v}+\bar{\rho} \operatorname{Src} . q_{v} \\
& \frac{\partial \bar{\rho} q_{x}}{\partial t}=\text { Adv } \cdot q_{x}+\text { Turb. } q_{x}+\bar{\rho} \operatorname{Src} . q_{x}+\bar{\rho} \text { Fall } \cdot q_{x}
\end{aligned}
$$

where $q_{x}$ is the representative mixing ratio of $q_{c}, q_{r}, q_{i}, q_{s} a n d q_{g}$, and "Adv.", "Turb." and "Fall." represent time changes due to advection, turbulent mixing, and fall out, respectively. All sources and sinks of variables are included in the "Src." term. The microphysical processes implemented in the model are described in Fig.5. Radiation of cloud is not included.

Turbulence is also an important physical process in the cloud model. Parameterizations of the subgrid-scale eddy motions in CReSS are one-order closure of the Smagorinsky (1963) [11] and the 1.5 order closer with turbulent kinetic energy (TKE). In the latter parameterization, the prognostic equation of TKE will be used.

CReSS implemented the surface process formulated by a bulk method. In this process, the surface sensible flux $H_{S}$ and latent heat flux $L E$ are formulated as

$$
\begin{aligned}
& H_{S}=-\rho_{a} C_{p} C_{h}\left|V_{a}\right|\left(T_{a}-T_{G}\right), \\
& L E=-\rho_{a} L C_{h}\left|V_{a}\right| \beta\left[q_{a}-q_{v s}^{*}\left(T_{G}\right)\right],
\end{aligned}
$$




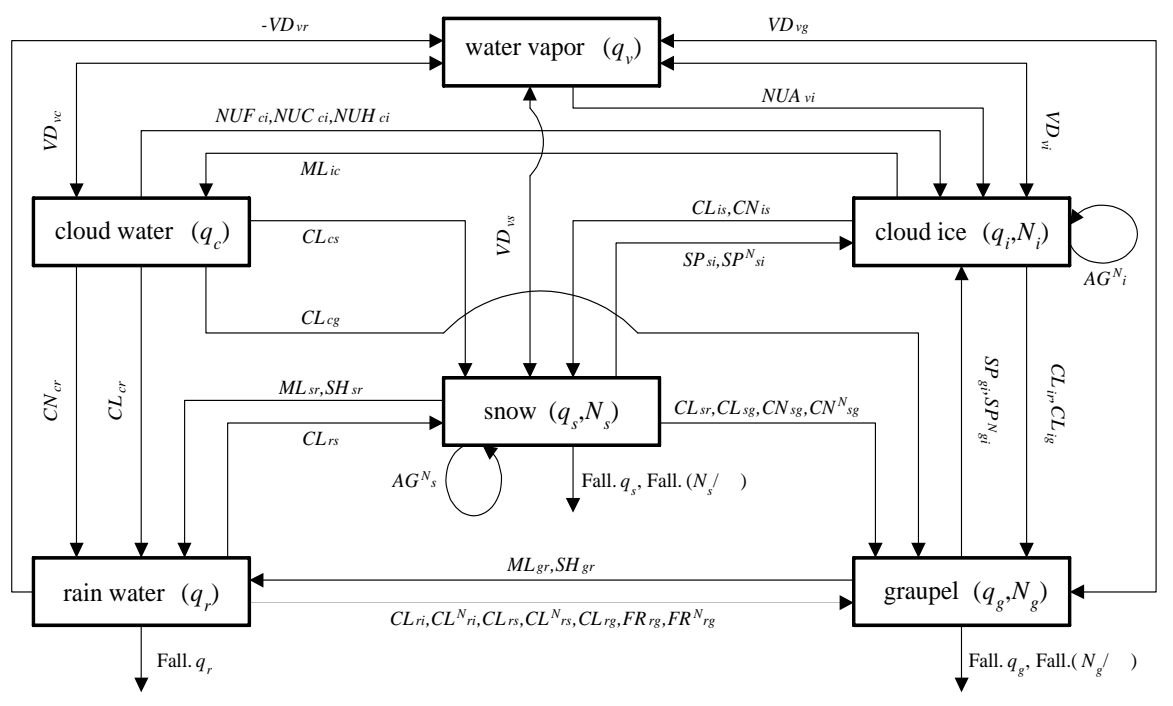

Fig. 5. Diagram describing of water substances and cloud microphysical processes in the bulk model.

where " $a$ " indicates the lowest layer of the atmosphere and " $G$ " the surface. The coefficient of $\beta$ is the evapotranspiration efficiency and $L$ is the latent heat of evaporation. The surface temperature of the ground $T_{G}$ is calculated by the $n$-layers ground model. The momentum fluxes $\left(\tau_{x}, \tau_{y}\right)$ are

$$
\begin{aligned}
& \tau_{x}=\rho_{a} C_{m}\left|V_{a}\right| u_{a}, \\
& \tau_{y}=\rho_{a} C_{m}\left|V_{a}\right| v_{a} .
\end{aligned}
$$

The bulk coefficients $C_{h}$ and $C_{m}$ are formulated by the scheme of Louis et al. (1981) [12].

\section{$3 \quad$ Dry Model Experiments}

In the development of CReSS, we tested it with respect to several types of phenomena. In a dry atmosphere, the mountain waves and the Kelvin-Helmholtz billows were chosen to test CReSS.

The numerical experiment of Kelvin-Helmholtz billows was performed in a two-dimensional geometry with a grid size of $20 \mathrm{~m}$. The profile of the basic flow was the hyperbolic tangent type. Stream lines of $u$ and $w$ components (Fig.6) show a clear cats eye structure of the Kelvin-Helmholtz billows. This result is very similar to that of Klaassen and Peltier (1985) [13]. The model also simulated the overturning of potential temperature associated with the billows (Fig.7). This result shows the model works correctly with a grid size of a few tens meters as far as in the dry experiment. 


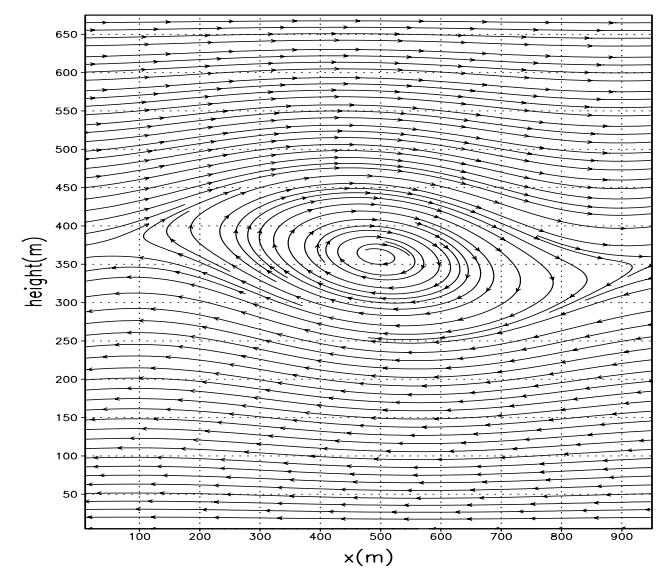

Fig. 6. Stream lines of the Kelvin-Helmholtz billow at 240 seconds from the initial simulated in the two-dimensional geometry.

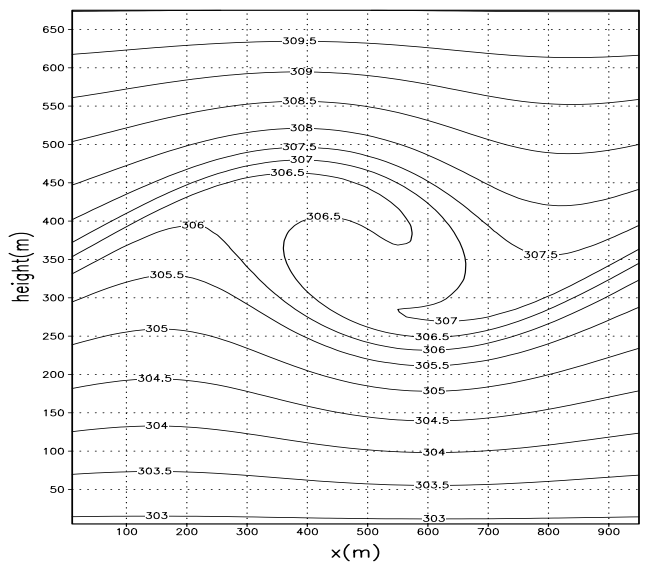

Fig. 7. Same as Fig.6, but for potential temperature.

In the experiment of mountain waves, we used a horizontal grid size of $400 \mathrm{~m}$ in a three-dimensional geometry. A bell-shaped mountain with a height of $500 \mathrm{~m}$ and with a half-width of $2000 \mathrm{~m}$ was placed at the center of the domain. The basic horizontal flow was $10 \mathrm{~m} \mathrm{~s}^{-1}$ and the buoyancy frequency was $0.01 \mathrm{~s}^{-1}$. The result (Fig.8) shows that upward and downwindward propagating mountain waves developed with time. The mountain waves pass through the downwind boundary. This result is closely similar to that obtained by other models as well as that predicted theoretically.

These results of the dry experiments showed that the fluid dynamics part and the turbulence parameterization of the model worked correctly and realistic behavior of flow were simulated. 


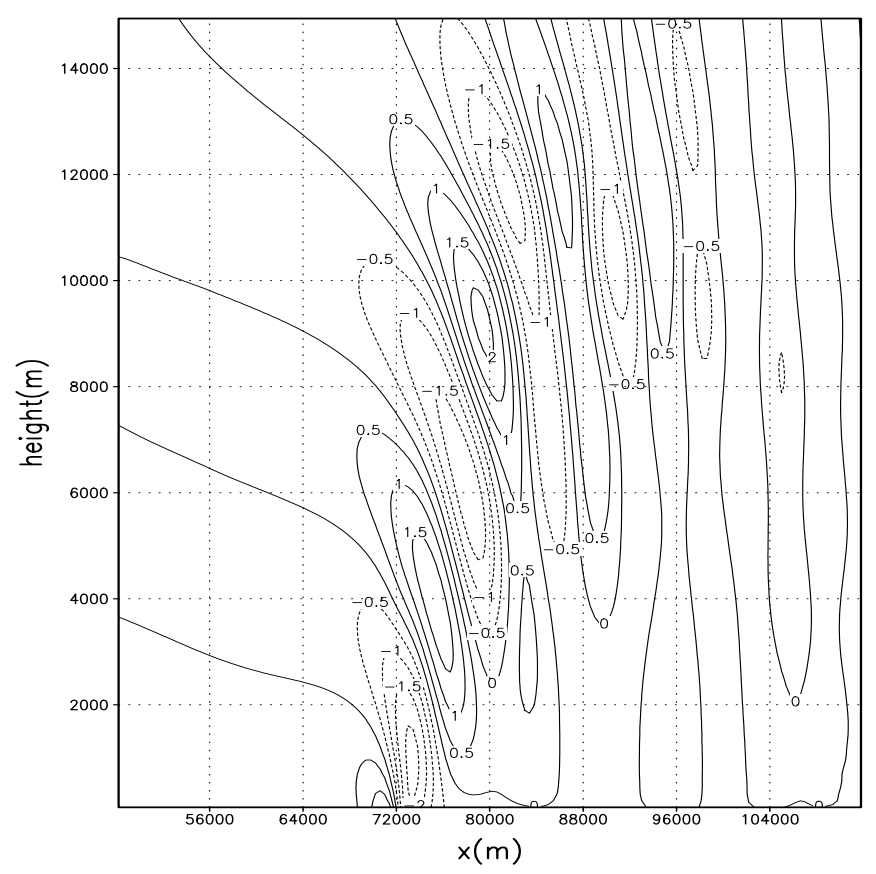

Fig. 8. Vertical velocity at 9000 seconds from the initial obtained by the mountain wave experiment.

\section{Simulation of Tornado within a Supercell}

In a simulation experiment of a moist atmosphere, we chose a tornado-producing supercell observed on 24 September 1999 in the Tokai District of Japan. The simulation was aiming at resolving the vortex of the tornado within the supercell.

Numerical simulation experiments of a supercell thunderstorm which has a horizontal scale of several tens kilometers using a cloud model have been performed during the past 20 years (Wilhelmson and Klemp, 1978 [14]; Weisman and Klemp, 1982, 1984 [15], [16]). Recently, Klemp and Rotunno (1983) [17] attempted to increase horizontal resolution to simulate a fine structure of a meso-cyclone within the supercell. It was still difficult to resolve the tornado. An intense tornado occasionally occurs within the supercell thunderstorm. The supercell is highly three-dimensional and its horizontal scale is several tens kilometer. A large domain of order of $100 \mathrm{~km}$ is necessary to simulate the supercell using a cloud model. On the other hand, the tornado has a horizontal scale of a few hundred meters. The simulation of the tornado requires a fine resolution of horizontal grid spacing of order of $100 \mathrm{~m}$ or less. In order to simulate the supercell and the associated tornado by a cloud model, a huge memory and high speed CPU are indispensable.

To overcome this difficulty, Wicker and Wilhelmson (1995) [18] used an adaptive grid method to simulate tornado-genesis. The grid spacing of the fine mesh 
was $120 \mathrm{~m}$. They simulated a genesis of tornadic vorticity. Grasso and Cotton (1995) [19] also used a two-way nesting procedure of a cloud model and simulated a tornadic vorticity. These simulations used a two-way nesting technique. Nesting methods include complication of communication between the coarsegrid model and the fine-mesh model through the boundary. On the contrary, the present research do not use any nesting methods. We attempted to simulate both the supercell and the tornado using the uniform grid. In this type of simulation, no complication of the boundary communication. The computational domain of the present simulation was about $50 \times 50 \mathrm{~km}$ and the grid spacing was $100 \mathrm{~m}$. The integration time was about 2 hours.

The basic field was give by a sounding at Shionomisaki, Japan at 00 UTC, 24 September 1999 (Fig.9). The initial perturbation was given by a warm thermal bubble placed near the surface. It caused an initial convective cloud.

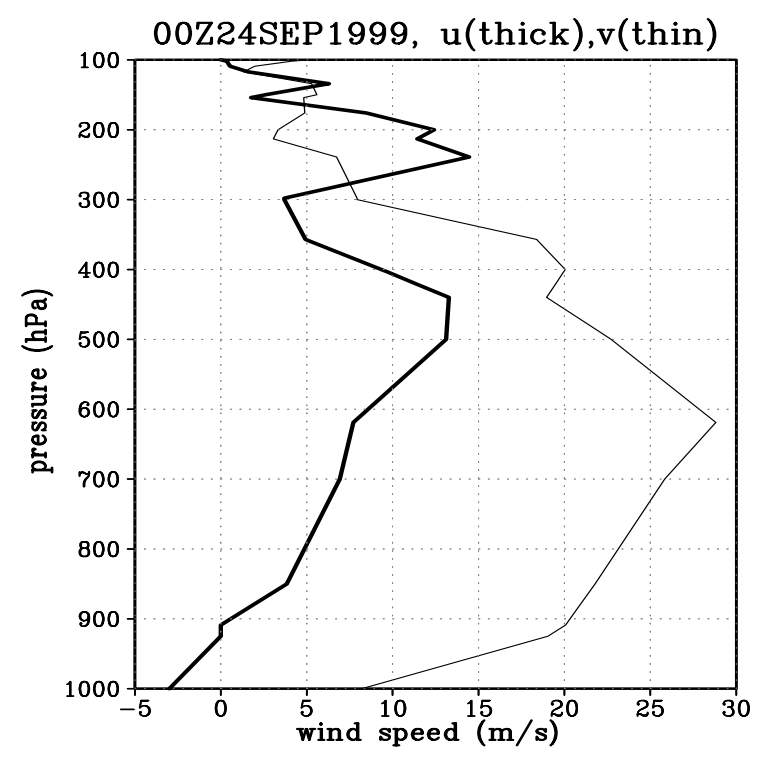

Fig. 9. Vertical profiles of zonal component (thick line) and meridional component (thin line) observed at Shionomisaki at 00 UTC, 24 September 1999.

After one hour from the initial time, a quasi-stationary super cell was simulated by CReSS (Fig.10). The hook-shaped precipitation area and the bounded weak echo region (BWER) which are characteristic features of the supercell were formed in the simulation. An intense updraft occurred along the surface flanking line. At the central part of BWER or of the updraft, a tornadic vortex was formed at 90 minutes from the initial time.

The close view of the central part of the vorticity (Fig.11) shows closed contours. The diameter of the vortex is about $500 \mathrm{~m}$ and the maximum of vorticity is about $0.1 \mathrm{~s}^{-1}$. This is considered to be corresponded to the observed tornado. 


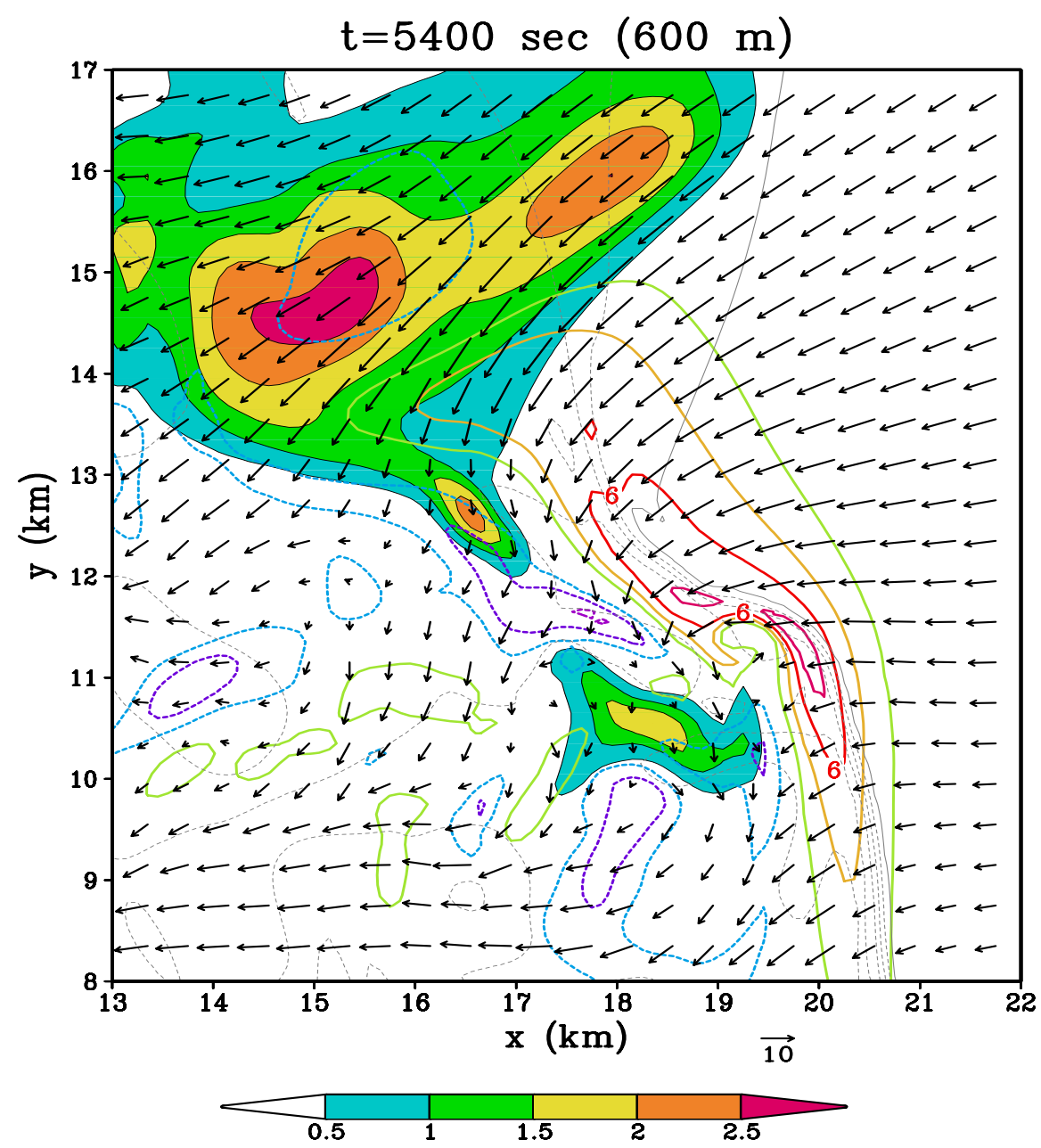

Fig. 10. Horizontal display at $600 \mathrm{~m}$ of the simulated supercell at 5400 seconds from the initial. Mixing ratio of rain (gray scales, $\mathrm{g} \mathrm{kg}^{-1}$ ), vertical velocity (thick lines, $\mathrm{m} \mathrm{s}^{-1}$ ), the surface potential temperature at $15 \mathrm{~m}$ (thin lines, $\mathrm{K}$ ) and horizontal velocity vectors.

The pressure perturbation (Fig.12) also shows closed contours which corresponds to those of the vorticity. This indicates that the flow of the vortex is in the cyclostrophic balance. The vertical cross section of the vortex (Fig.13) shows that the axis of the vorticity and the associated pressure perturbation is inclined to the left hand side and extends to a height of $2 \mathrm{~km}$. At the center of the vortex, the downward extension of cloud is simulated.

While this is a preliminary result of the simulation of the supercell and tornado, some characteristic features of the observation were simulated well. The important point of this simulation is that both the supercell and the tornado 
were simulated in the same grid size. The tornado was produced purely by the physical processes formulated in the model. A detailed analysis of the simulated data will provide an important information of the tornado-genesis within the supercell.

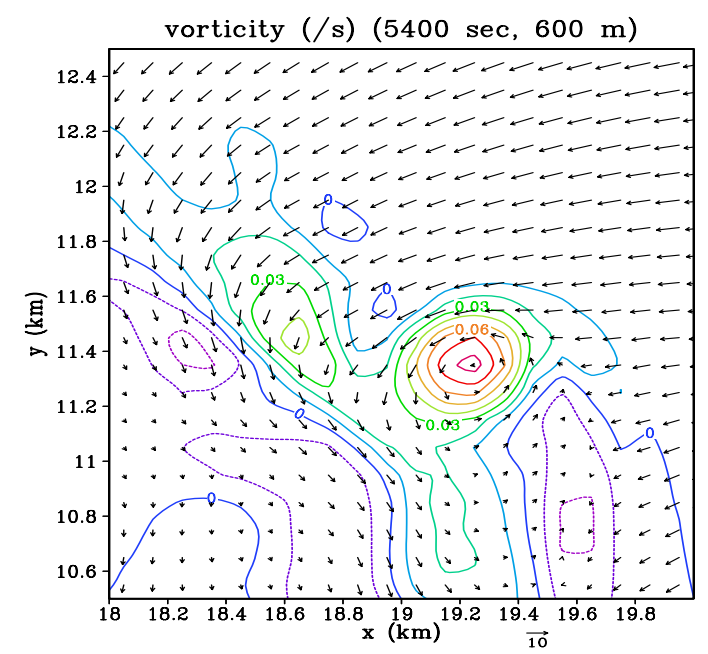

Fig. 11. Close view of the simulated tornado within the supercell. The contour lines are vorticity $\left(\mathrm{s}^{-1}\right)$ and the arrows are horizontal velocity. The arrow scale is shown at the bottom of the figure.

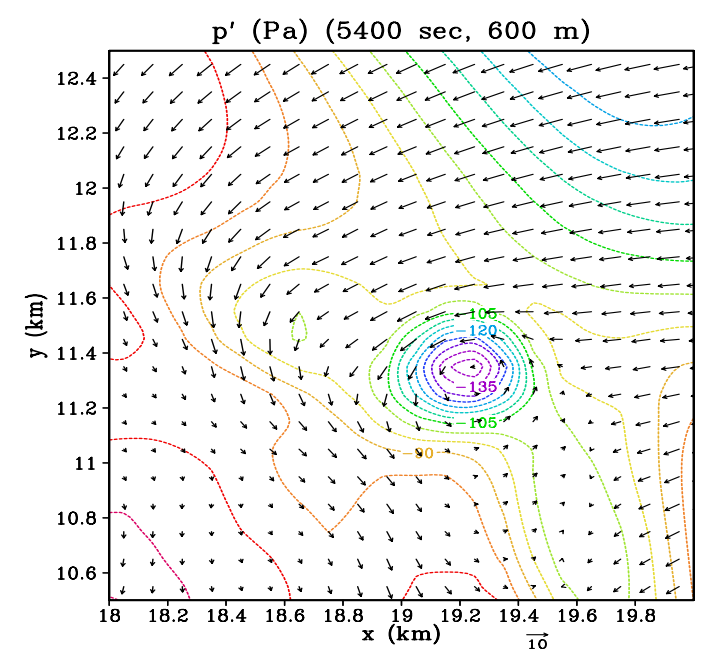

Fig. 12. Same as Fig.11, but for pressure perturbation. 


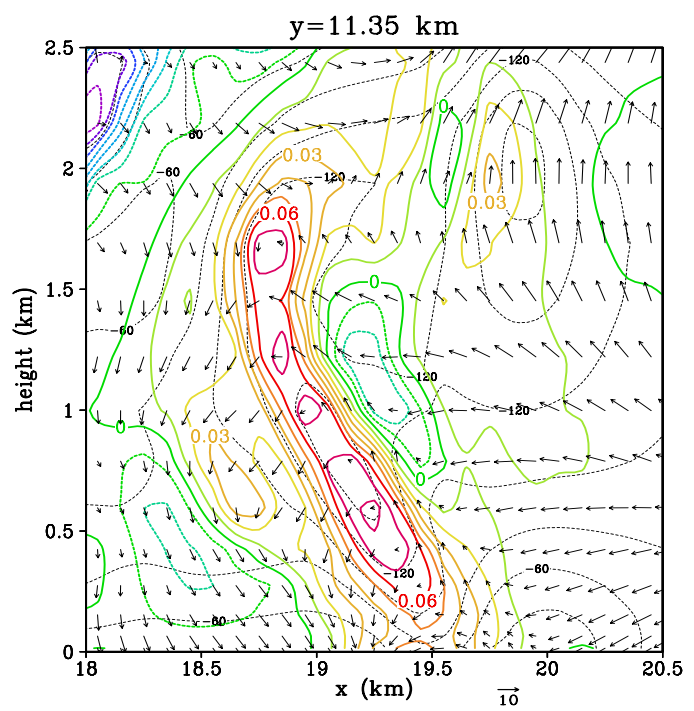

Fig. 13. Vertical cross section of the simulated tornado. Thick lines are vorticity $\left(\mathrm{s}^{-1}\right)$, dashed lines are pressure perturbation and arrows are horizontal velocity.

\section{Simulation of Squall Line}

A squall line is a significant mesoscale convective system. It is usually composed of an intense convective leading edge and a trailing stratiform region. An intense squall line was observed by three Doppler radars on 16 July 1998 over the China continent during the intensive field observation of GAME / HUBEX (the GEWEX Asian Monsoon Experiment / Huaihe River Basin Experiment). The squall line extended from the northwest to the southeast with a width of a few tens kilometers and moved northeastward at a speed of $11 \mathrm{~m} \mathrm{~s}^{-1}$. Radar observation showed that the squall line consisted of intense convective cells along the leading edge. Some of cells reached to a height of $17 \mathrm{~km}$. The rear-inflow was present at a height of $4 \mathrm{~km}$ which descended to cause the intense lower-level convergence at the leading edge. After the squall line passed over the radar sites, a stratiform precipitation was extending behind the convective leading edge.

The experimental design of the simulation experiment using CReSS is as follows. Both the horizontal and vertical grid sizes were $300 \mathrm{~m}$ within a domain of $170 \mathrm{~km} \times 120 \mathrm{~km}$. Cloud microphysics was the cold rain type. The boundary condition was the wave-radiating type. An initial condition was provided by a dual Doppler analysis and sounding data. The inhomogeneous velocity field within the storm was determined by the dual Doppler radar analysis directly while that of outside the storm and thermodynamic field were provided by the sounding observation. Mixing ratios of rain, snow and graupel were estimated from the radar reflectivity while mixing ratios of cloud and ice were set to be zero at the initial. A horizontal cross section of the initial field is shown in Fig.14. 


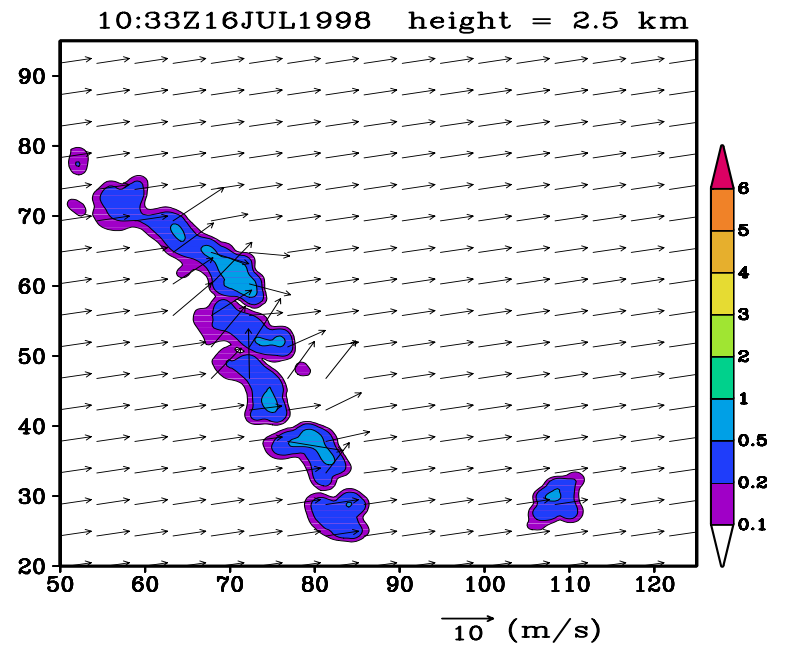

Fig. 14. Horizontal cross section of the initial field at a height of $2.5 \mathrm{~km}$ at 1033 UTC, 16 July 1998. The color levels mixing ratio of rain $\left(\mathrm{g} \mathrm{kg}^{-1}\right)$. Arrows show the horizontal velocity obtained by the dual Doppler analysis and sounding.
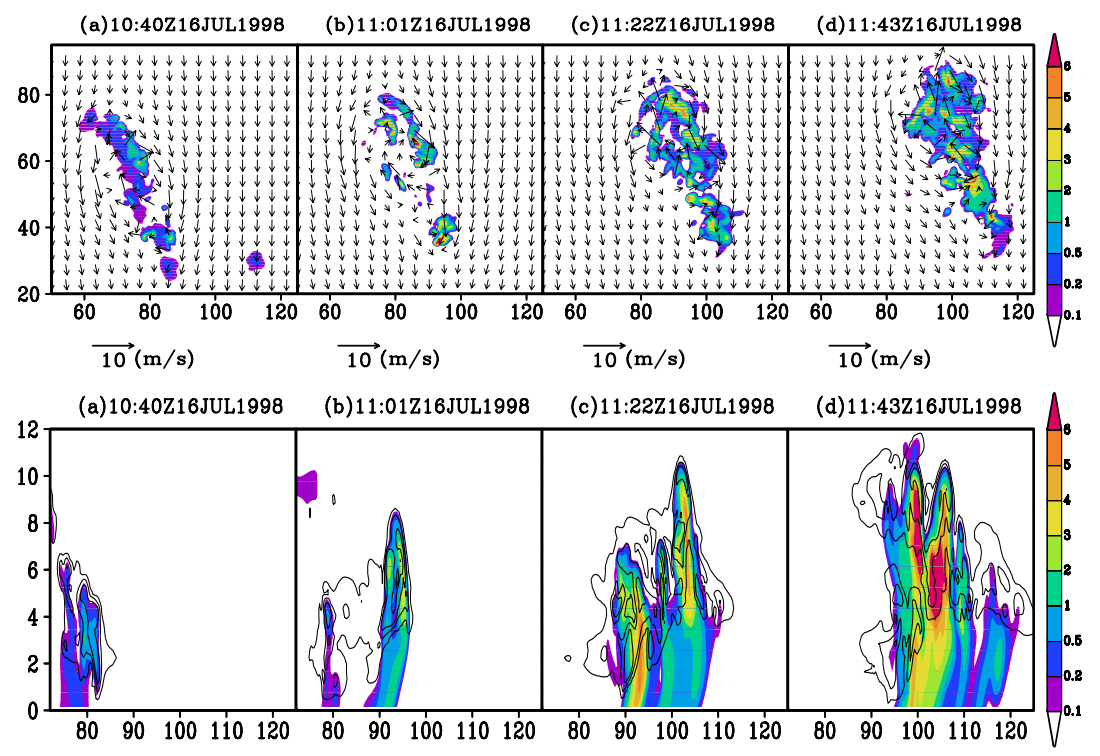

Fig. 15. Time series of horizontal displays (upper row) and vertical cross sections (lower row) of the simulated squall line. Color levels indicate total mixing ratio $\left(\mathrm{g} \mathrm{kg}^{-1}\right)$ of rain, snow and graupel. Contour lines indicate total mixing ratio $\left(0.1,0.5,1,2 \mathrm{~g} \mathrm{~kg}^{-1}\right)$ of cloud ice and cloud water. Arrows are horizontal velocity.

The simulated squall line extending from the northwest to the southeast moved northeastward (Fig.15). The convective reading edge of the simulated 
squall line was maintained by the replacement of new convective cells and moved to the northeast. This is similar to the behavior of the observed squall line. Convective cells reached to a height of about $14 \mathrm{~km}$ with large production of graupel above the melting layer. The rear-inflow was significant as the observation. A stratiform region extended with time behind the leading edge. Cloud extended to the southwest to form a cloud cluster.

The result of the simulation experiment showed that CReSS successfully simulated the development and movement of the squall line.

\section{Summary and Future Plans}

We are developing the cloud resolving numerical model CReSS for numerical experiments and simulations of clouds and storms. Parallel computing is indispensable for a large-scale simulations. In this paper, we described the basic formulations and important characteristics of CReSS. We also showed some result of the numerical experiments: the Kelvin-Helmholtz billows, the mountain waves, the tornado within the supercell and the squall line. These results showed that the CReSS has a capability to simulate thunderstorms and related phenomena.

In the future, we will make CReSS to include detailed cloud microphysical processes which resolve size distributions of hydrometeors. The parameterization of turbulence is another important physical process in cloud. The large eddy simulation is expected to be used in the model. We will develop CReSS to enable the two-way nesting within a coarse-grid model for a simulation of a real weather system. Four-dimensional data assimilation of Doppler radar is also our next target. Because initial conditions are essential for a simulation of mesoscale storms.

CReSS is now open for public and any users can download the source code and documents from the web site at "http://www.tokyo.rist.or.jp/CReSS_Fujin" (in Japanese) and can use for numerical experiments of cloud-related phenomena. CReSS has been tested on a several computers: HITACHI SR2201, HITACHI SR8000, Fujitsu VPP5000, NEC SX4. We expect that CReSS will be performed on the Earth Simulator and make a large-scale parallel computing to simulate a details of clouds and storms.

\section{Acknowledgements}

This study is a part of a project led by Professor Kamiya, Aichi Gakusen University. The project is supported by the Research Organization for Information Science and Technology (RIST). The simulations and calculations of this work were performed using HITACHI S3800 super computer and SR8000 computer at the Computer Center, the University of Tokyo and Fujitsu VPP5000 at the Computer Center, Nagoya University. The Grid Analysis and Display System (GrADS) developed at COLA, University of Maryland was used for displaying data and drawing figures. 


\section{References}

1. Klemp, J. B., and R. B. Wilhelmson, 1978: The simulation of three-dimensional convective storm dynamics. J. Atmos. Sci., 35, 1070-1096.

2. Ikawa, M., 1988: Comparison of some schemes for nonhydrostatic models with orography. J. Meteor. Soc. Japan, 66, 753-776.

3. Ikawa, M. and K. Saito, 1991: Description of a nonhydrostatic model developed at the Forecast Research Department of the MRI. Technical Report of the MRI, 28, 238pp.

4. Xue, M., K. K. Droegemeier, V. Wong, A. Shapiro and K. Brewster, 1995: Advanced Regional Prediction System, Version 4.0. Center for Analysis and Prediction of Storms, University of Oklahoma, 380pp.

5. Grell, G., J. Dudhia and D. Stauffer, 1994: A description of the fifth-generation of the Penn State / NCAR mesoscale model (MM5). NCAR Technical Note, 138pp.

6. Orlanski, I., 1976: A simple boundary condition for unbounded hyperbolic flows. J. Comput. Phys., 21, 251-269.

7. Lin, Y. L., R. D. Farley and H. D. Orville, 1983: Bulk parameterization of the snow field in a cloud model. J. Climate Appl. Meteor., 22, 1065-1092.

8. Cotton, W. R., G. J. Tripoli, R. M. Rauber and E. A. Mulvihill, 1986: Numerical simulation of the effects of varying ice crystal nucleation rates and aggregation processes on orographic snowfall. J. Climate Appl. Meteor., 25, 1658-1680.

9. Murakami, M., 1990: Numerical modeling of dynamical and microphysical evolution of an isolated convective cloud - The 19 July 1981 CCOPE cloud. J. Meteor. Soc. Japan, 68, 107-128.

10. Murakami, M., T. L. Clark and W. D. Hall 1994: Numerical simulations of convective snow clouds over the Sea of Japan; Two-dimensional simulations of mixed layer development and convective snow cloud formation. J. Meteor. Soc. Japan, $\mathbf{7 2}, 43-62$.

11. Smagorinsky, J., 1963: General circulation experiments with the primitive equations. I. The basic experiment. Mon. Wea. Rev., 91, 99-164.

12. Louis, J. F., M. Tiedtke and J. F. Geleyn, 1981: A short history of the operational PBL parameterization at ECMWF. Workshop on Planetary Boundary Layer Parameterization 25-27 Nov. 1981, 59-79.

13. Klaassen, G. P. and W. R. Peltier, 1985: The evolution of finite amplitude KelvinHelmholtz billows in two spatial dimensions. J. Atmos. Sci., 42, 1321-1339.

14. Wilhelmson, R. B., and J. B. Klemp, 1978: A numerical study of storm splitting that leads to long-lived storms. J. Atmos. Sci., 35, 1974-1986.

15. Weisman, M. L., and J. B. Klemp, 1982: The dependence of numerically simulated convective storms on vertical wind shear and buoyancy. Mon. Wea. Rev., 110, $504-520$.

16. Weisman, M. L., and J. B. Klemp, 1984: The structure and classification of numerically simulated convective storms in directionally varying wind shears. Mon. Wea. Rev., 112, 2478-2498.

17. Klemp, J. B., and R. Rotunno, 1983: A study of the tornadic region within a supercell thunderstorm. J. Atmos. Sci., 40, 359-377.

18. Wicker, L. J., and R. B. Wilhelmson, 1995: Simulation and analysis of tornado development and decay within a three-dimensional supercell thunderstorm. J. Atmos. Sci., 52, 2675-2703.

19. Grasso, L. D., and W. R. Cotton, 1995: Numerical simulation of a tornado vortex. J. Atmos. Sci, , 52, 1192-1203. 